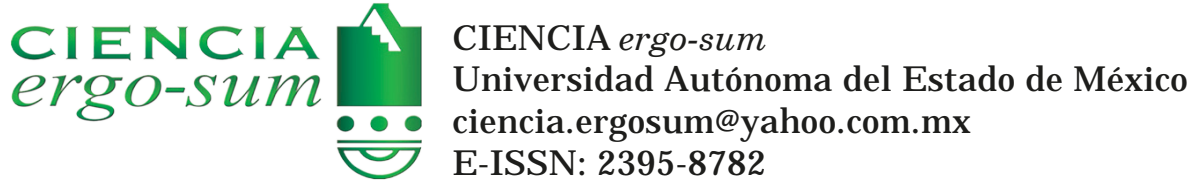

\title{
Calidad de plántula de árboles seleccionados de Leucaena esculenta en sistema agroforestal
}

Rodríguez-Ortiz, Gerardo; J osé-Hernández, Yesenia; Enríquez-del Valle, J osé Raymundo; Campos-Angeles, Gisela Virginia

Calidad de plántula de árboles seleccionados de Leucaena esculenta en sistema agroforestal

CIENCIA ergo-sum, vol. 28, núm. 1, marzo-junio 2021 |e113

Universidad Autónoma del Estado de México, México

Esta obra está bajo una Licencia Creative Commons Atribución-NoComercial-SinDerivar 4.0 Internacional.

Rodríguez-Ortiz, G., J osé-Hernández, Y., Enríquez-del Valle, J . R. y Campos-Angeles, G. V. (2021). Calidad de plántula de árboles seleccionados de Leucaena esculenta en sistema agroforestal. CIENCIA ergo-sum, 28(1). https://doi.org/10.30878/ces.v28n1a7 


\title{
Calidad de plántula de árboles seleccionados de Leucaena esculenta en sistema agroforestal
}

\section{Seedling quality from selected trees of Leucaena esculenta in agroforestry system}

\author{
Gerardo Rodriguez-Ortiz \\ Tecnológico Nacional de México, Campus Valle de Oaxaca \\ gerardo.rodriguez@voaxaca.tecnm.mx \\ (D) http://orcid.org/0000-0003-0963-8046 \\ Yesenia José-Hernández \\ Tecnológico Nacional de México, Campus Valle de Oaxaca. \\ joseyesenia93@gmail.com \\ (D) https://orcid.org/0000-0002-7750-2813 \\ José Raymundo Enríquez-del Valle \\ Tecnológico Nacional de México, Campus Valle de Oaxaca \\ jose.enriquez@voaxaca.tecnm.mx \\ (D) https://orcid.org/0000-0002-7700-3790 \\ Gisela Virginia Campos-Angeles \\ Tecnológico Nacional de México, Campus Valle de Oaxaca \\ gisela.campos@voaxaca.tecnm.mx \\ (1) https://orcid.org/0000-0003-2258-7124
}

\author{
Recepción: 02 de julio de 2018 \\ Aprobación: 06 de marzo de 2019
}

\section{Resumen}

En condiciones de invernadero se evaluó la calidad de plántulas de progenies selectas de Leucaena esculenta. En este sentido, durante 2017 se estudió el crecimiento de plantas en función de progenies de árboles y edad después de la siembra $(D D S)$ realizando análisis de varianza y pruebas de medias (Duncan, 0.05). Las progenies y los $D D S$ mostraron separadamente diferencias significativas $(p \leq 0.0001)$ en las variables morfológicas y área foliar. Los índices de calidad fueron diferentes entre árboles (progenies) y $D D S$, donde el árbol 9 tuvo el valor mayor $(4.54 \pm 3.03)$ en el Índice de Dickson. Con los indicadores de calidad de planta se concluye que las progenies del árbol 7 fueron las de mejor calidad con valores mayores de las variables morfológicas.

Palabras clave: área foliar, índice de calidad de planta, semillas ortodoxas.

\section{Abstract}

Under greenhouse conditions, seedlings quality of Leucaena esculenta selected progenies were evaluated. In this sense, in 2017 the plant growth was analyzing according to tree progenies and age after sowing, $A A S$, using analysis of variance and mean tests (Duncan, 0.05 ). The progenies and $A A S$ showed separately significant differences $(p \leq 0.0001)$ in morphological variables and leaf area. Quality indexes showed significant differences between trees (progenies) and $A A S$, where tree 9 had the highest Dickson Index (4.54 \pm 3.03$)$. According to plant quality indexes, it concludes that seedlings from tree 7 were the best with the highest values of the morphological variables. KEYWORDS: leaf area, plant quality index, orthodox seeds.

\section{INTRODUCCIÓN}

Una de las especies arbóreas utilizadas para los sistemas agroforestales de ladera es Leucaena esculenta (Moc. et Sessé ex Dc.) Benth., que en México se le conoce como guaje. Sin embargo, para la propagación de grandes cantidades de plantas hay limitaciones debido a la latencia de las semillas causada por la presencia de una testa gruesa dura e impermeable (González et al., 2009; Sánchez y Ramírez-Villalobos, 2006; Ruiz et al., 2007) que no permite 
la entrada del oxígeno y agua para que ocurra la imbibición y se inicie el crecimiento del embrión, por lo que el porcentaje de las semillas que germinan es bajo (Poulsen, 2000).

La conservación, estudio y gestión sostenible de los recursos genéticos forestales es un tema fundamental para la humanidad, ya que garantizaría que las generaciones actuales y futuras continúen obteniendo beneficios de los bosques, en general, y de sus componentes, en particular (Mallén-Rivera, 2014). La posibilidad de colectar germoplasma (frutos) (Jardel, 2006) de varios árboles identificados como sobresalientes, y procedentes de masas forestales naturales, es el punto de partida para propagar la especie y conservar una amplia base genética, así como una estrategia para aumentar los rendimientos de plantaciones forestales, ya que Viveros-Viveros et al. (2006) compararon durante 24 meses el crecimiento de nueve procedencias de Pinuspseudostrobus Lindl. típico y dos procedencias de P. pseudostrobus var. apulcensis Mart. que se establecieron en una localidad del estado de Michoacán. Encontraron que el $P$. pseudostrobus Lindl. típico tuvo en promedio mayor crecimiento que el $P$.pseudostrobus var. apulcensis, pero también diferencias notables de altura entre procedencias de la misma especie. En la vegetación silvestre se utiliza el método de selección individual, lo cual implica elegir los individuos con base únicamente en su fenotipo, pero con la consideración de que es un buen reflejo del genotipo (Zobel y Talbert, 1988). Algunos criterios para aplicar la selección, es que los árboles deben ser sanos, libres de plagas y enfermedades, ser dominantes y codominantes.

Se considera que la propagación mediante semillas es la forma más barata y práctica (Torres et al., 2002), aunque éstas presentan dormancia física por la impermeabilidad de sus cubiertas seminales, en coincidencia con lo descrito por Willan (2000) y por Kozlowski y Pallardy (2002) para las especies arbóreas del trópico seco y con lo informado en Albizia lebbeck (L.) Benth (Navarro, 2003) en donde reporta para Leucaena leucocephala cv. Cunningham hasta un $80 \%$ de dormancia.

En la actualidad, existen métodos más sofisticados para determinar la calidad de las plantas, fundamentados en las características morfológicas y fisiológicas (Toral, 1997; Haase, 2007), los cuales son indicadores de su capacidad para sobrevivir y crecer en las condiciones climáticas y edáficas del sitio de plantación, que depende, en gran medida, de la calidad genética del germoplasma utilizado y de las condiciones proporcionadas a las plantas durante su producción en vivero: sustratos, envases, nutrición, riego, sanidad y manejo integral de dichos factores (Ramírez y Rodríguez, 2004).

Es preciso identificar las características positivas de la planta para su posterior utilización y manejo en condiciones de campo. Para determinar la calidad de la planta se consideran criterios morfológicos, que son los más utilizados debido a la facilidad con la que se evalúan. Entre esos criterios destacan la altura del tallo, el diámetro al cuello y la cantidad de biomasa, que a su vez permite estimar el índice de robustez (altura/diámetro) y la relación biomasa parte aérea/raíz. De acuerdo con Rodríguez (2008), la biomasa total de la planta obtenida en invernadero y las proporciones que de esta biomasa corresponden a tallo, hojas y raíz son un indicador del desarrollo y capacidad de la planta para adaptarse a campo dado que en tres meses y medio están listas para llevarlas al campo, una vez que hayan alcanzado una altura promedio de $30 \mathrm{~cm}$, ya que si se dejan más tiempo en invernadero, el sistema radical comienza a obstruirse y aumenta el porcentaje de mortalidad en el campo. De acuerdo con Prieto et al. $(2003,2009)$, el índice de calidad de Dickson y la relación altura: longitud de raíz son indicadores que predicen el éxito de la plantación. Prieto et al. $(2003,2009)$ también comentan que debe existir equilibrio y proporción entre la parte aérea y el sistema radical de las plantas. Por ello se planteó el objetivo de evaluar, en condiciones de invernadero, la calidad de plántulas de Leucaena esculenta obtenidas de semillas que son colectadas de diversos árboles semilleros, los cuales se encuentran establecidos en un sistema agroforestal de ladera.

\section{Materiales y MÉtodos}

\section{1. Localización del área de estudio}

El estudio se realizó en un invernadero ubicado en terrenos del Instituto Tecnológico del Valle de Oaxaca (ITVO), en Valles Centrales de Oaxaca, ubicado entre las coordenadas $17^{\circ} 01^{\prime} 07.9^{\prime \prime} \mathrm{LN}$ y $096^{\circ} 45^{\prime} 56.9^{\prime \prime} \mathrm{LO}$, con una 
altitud de $1566 \mathrm{~m}$. El clima en la zona es templado con lluvias en verano, con temperatura promedio de $20.4^{\circ} \mathrm{C}$ y $669 \mathrm{~mm}$ de precipitación anual. Durante los meses marzo-abril se colectaron las semillas-progenies de nueve árboles que crecen en el sistema agroforestal en terreno de ladera dentro del mismo instituto.

El ambiente agroforestal está ubicado en exposición norte de una ladera con 15\% de pendiente original, donde se plantaron en 2006 los árboles cada $1.2 \mathrm{~m}$ en la hileras ubicadas en curvas a nivel espaciadas cada $6.5 \mathrm{~m}$. En el transcurso de los años se formaron terrazas con pendiente de $2 \%$; en tales terrazas se establecieron gramíneas y en algunos años se cultivó maíz. Transcurridos 11 años de establecida la plantación, los árboles tenían de 6 a $12 \mathrm{~m}$ de altura, y 10 a $20 \mathrm{~cm}$ de diámetro normal. De una población de 87 árboles, se seleccionaron 9 para colectar semilla, ya que éstos sobresalían por tener $12 \mathrm{~m}$ o más de altura y $20 \mathrm{~cm}$ o más de diámetro, de apariencia sanos y buena conformación.

\section{2. Índices de calidad de planta}

Los métodos se basaron en normas establecidas por la International Seed Testing Association (ISTA, 2007). Las semillas de cada árbol se escarificaron sumergiéndolas por $30 \mathrm{~min}$ en agua a $100{ }^{\circ} \mathrm{C}$. Las semillas se germinaron en charolas de plástico de 49 cavidades de $37 \mathrm{~cm}^{3}$ usando sustrato compuesto de turba (40\%), vermiculita (30\%) y perlita (30\%). En cada cavidad de la charola se colocaron dos semillas a $0.5 \mathrm{~cm}$ de profundidad y se utilizaron 294 semillas por árbol.

En 2017 se realizó cada 15 días y durante tres meses un muestreo destructivo después de la siembra $(D D S)$ de tres plantas por progenie de las cuales se separaron hojas, ramas, raíz y tallo con la ayuda de una tijera para podar. Se registró la altura $(\mathrm{AT}, \mathrm{cm})$ desde el cuello de la raíz hasta el ápice de la planta; el diámetro del cuello de la raíz (DC, mm) se obtuvo con un vernier digital (Modelo 500-196-30, Alemania) con precisión $\pm 0.1 \mathrm{~mm}$; biomasa (g) en fresco y en seco de la raíz y de la parte aérea separando hojas, ramas, tallo. Para determinar los pesos secos, los diversos órganos de cada planta se colocaron por separado en bolsas de papel y se colocaron hasta obtener peso constante en una estufa de convección eléctrica marca Memmer a una temperatura constante de $70^{\circ} \mathrm{C}$. Transcurrido este tiempo los pesos se determinaron con una báscula digital (Modelo H-2716, Alemania).

Los índices de calidad de planta se obtuvieron de acuerdo con Rodríguez (2008) con las siguientes fórmulas:

$$
I E=\frac{A T}{D C}, I D=\frac{P S T}{\frac{A T}{D C}+\frac{P S A}{P S R}}, R A S=\frac{P S A}{P S R}, R A R=\frac{A T}{L R}
$$

Donde: $\mathrm{IE}=$ índice de esbeltez, ID = índice de calidad de Dickson, RAS = relación biomasa aérea-biomasa subterránea, $\mathrm{RAR}=$ relación parte aérea/raíz, $\mathrm{AT}=$ altura total $(\mathrm{cm}), \mathrm{DC}=$ diámetro al cuello de la raíz $(\mathrm{mm})$, PST $=$ peso seco total $(\mathrm{g})$, PSA $=$ peso seco parte aérea $(\mathrm{g}), \mathrm{PSR}=$ peso seco de raíz, $\mathrm{LR}=$ longitud de raíz $(\mathrm{cm})$.

\subsection{Análisis estadístico de datos}

El experimento se estableció de acuerdo con un diseño completamente aleatorio con arreglo factorial $(9 \times 6)$ y se evaluaron progenies de nueve árboles en seis fechas de muestreo después de la siembra $(D D S)$. La unidad experimental fue una planta con 18 repeticiones por árbol (repartidas en los $D D S$ ).

Los datos se integraron en una hoja de cálculo de Office Excel de Microsoft ${ }^{\circledR}$ donde se registró, especie, sustrato, tratamiento y mediciones obtenidas en los muestreos destructivos. Los datos de variables morfológicas y de crecimiento se sometieron a análisis de varianza y prueba de separación de medias (Duncan, $\alpha=0.05$ ). Las variables que no cumplieron los supuestos de normalidad y homogeneidad de varianzas se transformaron con $\log (x)$. Las rutinas de análisis se realizaron con el paquete estadístico SAS (Sistema de Análisis Estadístico) (SAS Institute Inc., 2004). 


\section{Resultados Y Discusión}

\section{1. Crecimiento de plántulas}

La importancia de propagar y establecer plantaciones con especies nativas es útil para diversificar la base productiva de bienes derivados del bosque. Relacionado con lo anterior, la propuesta de hacer una selección con fines de mejoramiento genético pretende agregar valor a los cultivos y mejorar su productividad (Gutiérrez, 2003). Durante el crecimiento de las plántulas de L. esculenta en invernadero, los análisis de varianza mostraron diferencias significativas y altamente significativas $(p \leq 0.05 ; \leq 0.01)$ para las progenies en la mayoría de variables, excepto en DC, mientras que para $D D S$ fue altamente significativa para todas las variables. Para el caso de las interacciones sólo tuvieron efectos significativos $(p \leq 0.05)$ en longitud de raíz, AF y RAR (cuadro 1 ). Este resultado es ligeramente más bajo que lo reportado por Santiago et al. (2007), quienes mencionan que el rango de altura de plantas de esta especie debe ser de 25 a $30 \mathrm{~cm}$. Existen resultados semejantes en especies perennes como Theobroma cacao L. y Coffea arabica L. (Sánchez et al., 2005); por otra parte, Wu et al. (2008) reportaron que en Sophora davidii Kom. ex. Pavol. sometidas a diversas condiciones de estrés de humedad, las diferencias en biomasa foliar y tallo sólo se presentaron en el tratamiento de estrés severo (riego diario a 15\%, de la capacidad de campo-CC, contenido de agua o humedad que es capaz de retener el suelo, mientras que las plantas sometidas a estrés moderado (riego diario a 30\% de la CC) no mostraron diferencias en comparación con el grupo control (riego diario a 45\% de la CC).

\section{CUADRO 1}

Resumen del análisis de varianza de las variables morfológicas evaluadas en progenies de árboles semilleros de Leucaena esculenta a diferentes días después de la siembra (DDS)

\begin{tabular}{|lrccccl}
\hline \multicolumn{7}{cl}{ Cuadrados medios y significancia } \\
\hline Fuente de variación & $\mathrm{Gl}$ & $\mathrm{AT}(\mathrm{cm})$ & $\mathrm{DC}(\mathrm{mm})$ & $\mathrm{LR}(\mathrm{cm})$ & $\mathrm{AF}\left(\mathrm{cm}^{2}\right)$ & $\mathrm{RAR}$ \\
Árbol & 8 & $26.2^{* *}$ & $21.7 \mathrm{~ns}$ & $3.3^{*}$ & $56.8^{*}$ & $0.09^{* *}$ \\
$D D S$ & 5 & $173.9^{* *}$ & $523.7^{* *}$ & $38.1^{* *}$ & $3509.3^{* *}$ & $0.38^{* *}$ \\
Árbol $\times D D S$ & 38 & $2.8 \mathrm{~ns}$ & $20.3 \mathrm{~ns}$ & $3.09^{* *}$ & $74.6^{* *}$ & $0.01^{*}$ \\
Error & 104 & 2.3 & 15.1 & 1.52 & 21.05 & 0.01 \\
Total & 155 & & & & & \\
\hline
\end{tabular}

Fuente: elaboración propia con base en los análisis.

Nota: $\mathrm{Gl}=$ grados de libertad, $\mathrm{AT}=$ altura total de la plántula, $\mathrm{DC}=$ diámetro al cuello de la raíz, $\mathrm{LR}=$ longitud de la raíz, $\mathrm{AF}=$ área foliar, $\mathrm{RAR}=$ relación altura-longitud de raíz, ${ }^{*}=$ valor de $\mathrm{F}$, significativo $(p \leq 0.05), * *=$ valor de $\mathrm{F}$, altamente significativo $(p \leq 0.001)$, $n s=$ valor de $\mathrm{F}$, no significativo $(p \leq 0.05)$.

La progenie del árbol 7 mostró la mayor altura $(13.83 \pm 2.61 \mathrm{~cm})$, seguido de la progenie del árbol $1(12 \pm 3.08 \mathrm{~cm})$. Ferrer et al. (2003) evaluaron el crecimiento de plántulas de Leucaena leucocephala (Lam.) de Wit y Pithecelobium dulce (Roxb.) Benth obteniendo valores medios de altura para L. leucocephala $(7.96 \mathrm{~cm})$, e inferiores a los encontrados en este estudio, aunque en dicho trabajo no se indicó el tiempo en el que se realizaron estas observaciones, por lo que se puede atribuir al intervalo de medición.

En cuanto al diámetro del cuello de la raíz (diámetro basal), las progenies de los árboles 7 y 4 mostraron valores similares $(p>0.05)$ de $24.1 \pm 6.7 \mathrm{~mm}$ durante los primeros días del experimento, lo cual se refleja en el índice de esbeltez y RAR (cuadros 1 y 2); sin embargo, las diferencias se encontraron al final del experimento (90 a 105 $D D S)$. Estadísticamente, los valores alcanzados en este estudio difieren de los documentados por Medina et al. (2007, 2011) para un periodo similar, en el cual el diámetro de cuello alcanzado fue de $9 \mathrm{~mm}$ para Leucaena leucocephala cv. Cunningham. 
El área foliar $(A F)$ fue significativa $(p \leq 0.05)$ en los $D D S$, entre progenies y la interacción Árbol $\times D D S$; este efecto fue semejante hasta el final del estudio (cuadro 1). A los 90 y 105 DDS la progenie del árbol 9 generó la mayor área foliar (cuadro 4). Guevara y Guenni (2007) reportan sobre el potencial de crecimiento de cuatro líneas de Leucaena leucocephala en donde describen diferencias de crecimiento.

La relación entre altura y la longitud de la raíz $(R A R)$ indica un alto crecimiento $0.82 \pm 0.15 \mathrm{~cm} \mathrm{~mm}^{-1}$, como el caso de la progenie 7 (cuadros 3 y 4), lo cual puede deberse a ajustes en la relación fuente-demanda de agua en las plántulas (Wullschleger et al., 2005); esto es contrario a lo que ha sido observado en plántulas caducifolias de Dalbergia sisso Roxb., que bajo condiciones de estrés hídrico moderado no mostraron diferencias sobre el crecimiento y biomasa de la raíz (Singh y Singh, 2006).

\section{2. Índices de calidad de plántula}

En este estudio el índice de calidad de Dickson (ID) fue significativamente diferente $(p<0.05)$ en todas las progenies, en los $D D S$ y en la interacción (cuadro 2). El árbol 6 presentó el mayor ID, $4.54 \pm 3.03$ (cuadro 4). Sáenz et al. (2010) encontraron un ID de 0.79 para (moringa) Moringa oleifera Lam., valor inferior al de guaje, por lo que puede comprobarse la mejor calidad de las plántulas estudiadas. Al respecto, Sáenz et al. (2010) concluyen sobre la relación directa entre el valor del índice y la sobrevivencia de las plantas; así, ID $<0.15$ significa problemas en el establecimiento para algunas especies. González et al. (1996) mencionan que es un índice desarrollado para evaluar distintas combinaciones de parámetros morfológicos. De este modo, los datos del presente trabajo indican que las plántulas de L. esculenta son de buena calidad.

El índice de esbeltez $(I E)$ no mostró diferencias significativas $(p>0.05)$ entre Árbol y la interacción Árbol $\times$ $D D S$ (cuadro 2); el promedio fue de $0.07 \pm 0.17$ (cuadro 4); sin embargo, la prueba de medias genera diferencias entre progenies y $D D S$. En un estudio realizado por Orozco et al. (2010) para el índice de esbeltez se obtuvieron valores mayores para (tepemezquite) Lysiloma divaricata (Jacq) y (guaje rojo) Leucaena leucocephala cv. 10.8 y 8.2 respectivamente. Santiago et al. (2007) recomiendan que mientras menor valor se obtenga en la relación altura/diámetro para especies tropicales será un indicador de un mayor vigor de la planta. Birchler et al. (1998) sugieren una baja equitatividad y por tanto baja robustez, ya que según los autores éste debe ser menor de seis para indicar una alta resistencia a la desecación, tal como lo encontrado en este estudio.

Las progenies que generaron mayor biomasa total fueron las originadas de los árboles 7 y $4(0.53 \pm 0.16$ y $0.51 \pm$ $0.16 \mathrm{~g} /$ árbol). La parte que más captura biomasa con un promedio de $0.36 \pm 0.12 \mathrm{~g} /$ árbol son las hojas, seguido de la raíz y tallo a los $105 D D S$ (gráficas 1 y 2). Este parámetro es un indicador del desarrollo de la planta y producción de biomasa en invernadero. Escobar-Sandoval et al. (2018) al estudiar la heredabilidad y correlaciones genéticas en Pinus patula mencionan que es posible utilizar algunas características morfológicas con fines de selección de manera independiente o combinada para aumentar el crecimiento y calidad de la madera. En dicho trabajo encontraron que todas las características de crecimiento y ramificación presentaron valores de heredabilidad de bajos a moderados, que es un indicador de un fuerte efecto ambiental en la expresión de las características. Así también, Ortiz et al. (2016) en dos localidades del estado de Veracruz seleccionaron fenotípicamente 40 árboles silvestres de Juglans pyriformis Liebm, de los cuales colectaron semillas para germinarlas y desarrollaron las plantas en vivero. Con base en el desempeño de las progenies, seleccionaron 20 árboles para colectar semillas, las cuales darán origen a plántulas de calidad. Mencionan obtener germoplasma a partir de una fuente identificada procedente de masas forestales naturales lo que repercute en que la colecta tenga base genética amplia.

Los valores de la RAS > 1.7 para las progenies (cuadros 3 y 4 ) es un indicador del desarrollo balanceado de la planta; este índice (RAS) fue significativamente diferente $(p>0.05)$ entre árboles, $D D S$ y la interacción Árbol $\times$ $D D S$, donde el árbol 8 tuvo el valor más bajo, $1.65 \pm 0.82$ (cuadro 4), lo que indica que la biomasa aérea es mayor que la subterránea. Estos resultados son similares a los encontrados por Noguera-Talavera et al. (2016) en la especie de moringa (Moringa oleifera Lam.) registrando una biomasa seca aérea y biomasa seca raíz (BSA/BSR) 1.8 a la 
semana 10, lo que indica que la biomasa aérea es mayor que la subterránea. Según Rodríguez (2008), este valor es indicador de una buena relación entre ambas partes y sugiere la existencia de un sistema radical suficiente para proveer agua y nutrimentos minerales a la parte aérea de la planta. Thompson (1985) asevera que el tamaño del sistema de raíz es importante cuando la planta crece en sitios en que la precipitación es escasa. En este sentido, García (2007) indica que una planta de buena calidad debe presentar características como un diámetro basal grande, un sistema radical fibroso y un bajo valor de la relación biomasa aérea y biomasa de raíz. Es conocido que la magnitud de la distribución de biomasa depende de factores ambientales y del genotipo (Olalde et al., 2000; Ramírez et al., 2007); las condiciones de manejo y temporalidad también influyen en la partición de biomasa, sobre todo en lo que corresponde al individuo (Chave et al., 2001; Rodríguez-Ortiz et al., 2011).

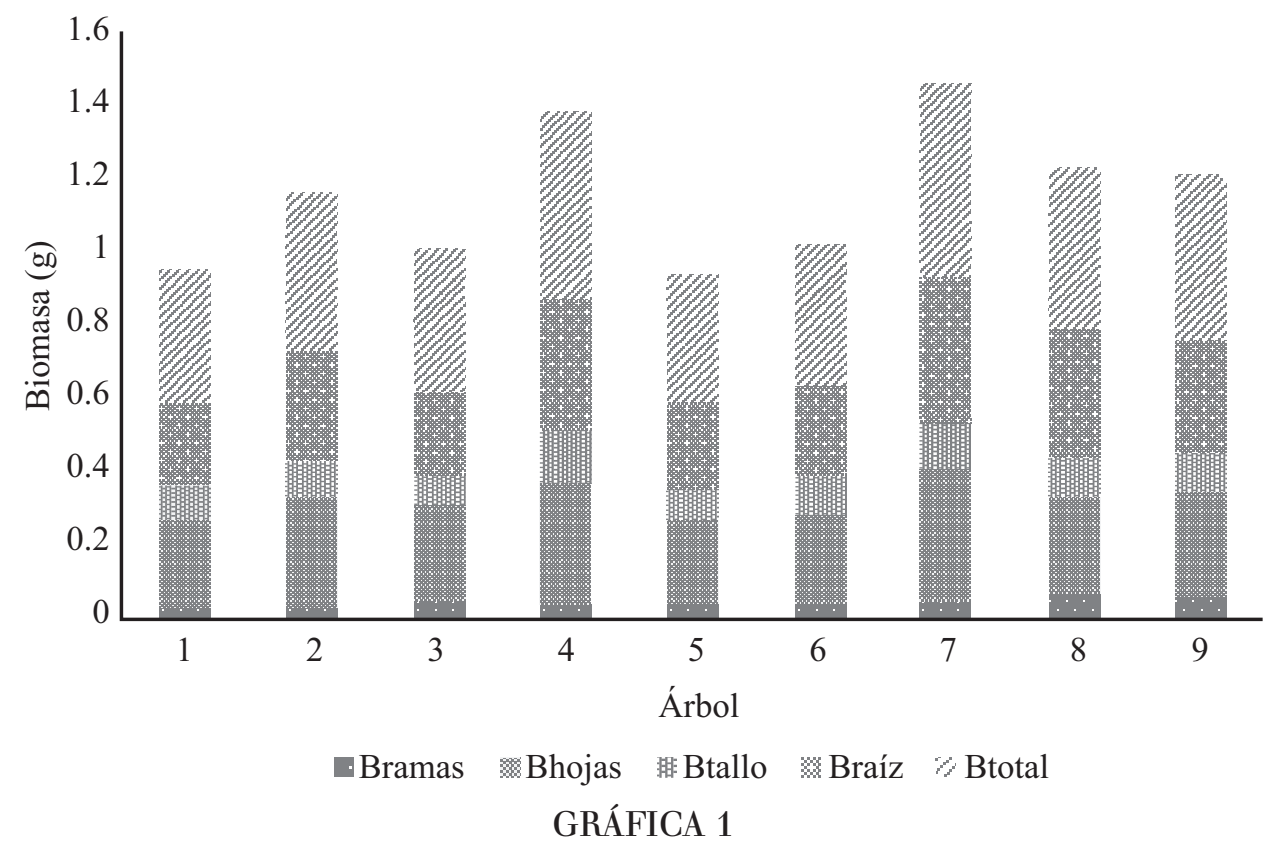

Biomasa (B) por componente en árboles de Leucaena esculenta

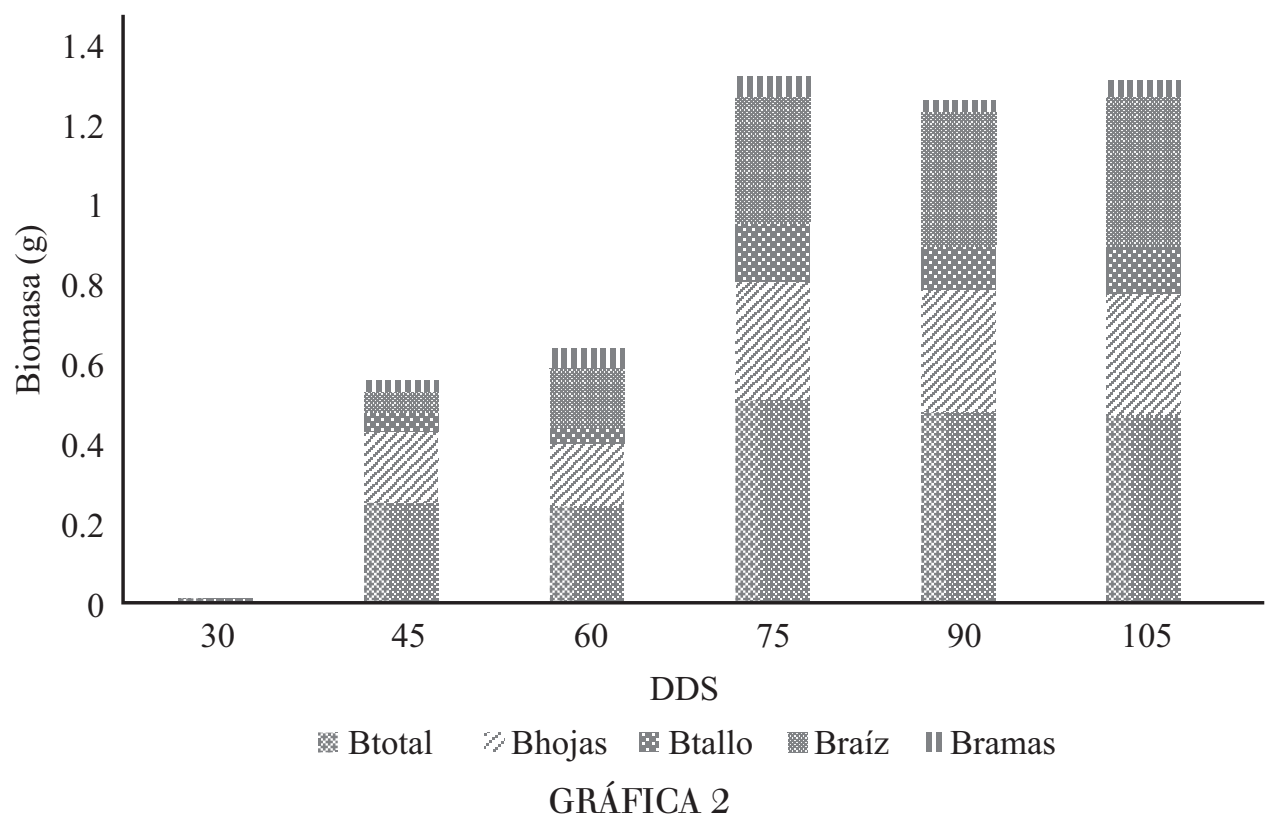

Biomasa (B) por componente de cada árbol y días después de su germinación (DDS) 


\section{CUADRO 2}

Resumen del análisis de varianza para índices morfológicos de calidad de planta de Leucaena esculenta

\begin{tabular}{|lccccc}
\hline \multicolumn{5}{c}{ Fuentes de variación, grados de libertad y cuadrados medios } \\
\hline Variable & Árbol & $D D S$ & Árbol $\times D D S$ & Error & Total \\
\hline & 8 & 5 & 38 & 104 & 155 \\
Índice de Dickson & $2.47^{*}$ & $30.2^{* *}$ & $1.8^{*}$ & 1.1 & \\
Índice de esbeltez & $0.06 \mathrm{~ns}$ & $0.18^{* *}$ & $0.02 \mathrm{~ns}$ & 0.03 \\
RAS & $3.9^{* *}$ & $60.9^{* *}$ & $2.3^{* *}$ & 0.83 \\
Biomasa foliar $(\mathrm{g})$ & $0.03^{* *}$ & $0.26^{* *}$ & $0.01^{* *}$ & 0.003 \\
Biomasa de tallo $(\mathrm{g})$ & $0.005^{* *}$ & $0.06^{* *}$ & $0.002^{* *}$ & 0.0009 \\
Biomasa de ramas $(\mathrm{g})$ & $3860.7^{* *}$ & $75466.5^{* *}$ & $2929.7^{* *}$ & 1177.6 \\
Biomasa de raíz $(\mathrm{g})$ & $0.06^{* *}$ & $0.06^{* *}$ & $0.03^{* *}$ & 0.009 \\
Biomasa total $(\mathrm{g})$ & $0.06^{* *}$ & $0.62^{* *}$ & $0.02^{* *}$ & 0.01 & \\
\hline
\end{tabular}

Fuente: elaboración propia con base en los análisis.

Nota: RAS = relación biomasa parte aérea y parte subterránea, $D D S=$ días después de la siembra, $*=$ Valor de $\mathrm{F}$, significativo $(p \leq 0.05)$, $* *=$ valor de $\mathrm{F}$, altamente significativo $(p \leq 0.001)$, ns = valor de $\mathrm{F}$, no significativo $(p>0.05)$.

\section{CUADRO 3}

Características de árboles de Leucaena esculenta de diferentes progenies, transcurridos 105 días de crecimiento en invernadero

\begin{tabular}{|lcllll|}
\hline DDS & AF $\left(\mathrm{cm}^{2}\right)$ & RAS & ID & \multicolumn{1}{c}{ RAR } & \multicolumn{1}{c}{ IE } \\
\hline 30 & $2.92 \pm 1.62 \mathrm{~d}$ & $5.42 \pm 2.62 \mathrm{a}$ & $5.82 \pm 2.57 \mathrm{a}$ & $0.52 \pm 0.19 \mathrm{~d}$ & $0.06 \pm 0.19 \mathrm{c}$ \\
45 & $2.83 \pm 2.41 \mathrm{~d}$ & $1.91 \pm 0.76 \mathrm{~b}$ & $2.92 \pm 0.67 \mathrm{c}$ & $0.57 \pm 0.11 \mathrm{dc}$ & $0.06 \pm \mathrm{c}$ \\
60 & $7.93 \pm 3.94 \mathrm{c}$ & $1.75 \pm 0.70 \mathrm{cb}$ & $3.18 \pm 0.73 \mathrm{c}$ & $0.60 \pm 0.13 \mathrm{c}$ & $0.07 \pm 0.35 \mathrm{a}$ \\
75 & $18.68 \pm 6.94 \mathrm{~b}$ & $1.52 \pm 0.52 \mathrm{cbd}$ & $3.08 \pm 0.61 \mathrm{c}$ & $0.77 \pm 0.13 \mathrm{ba}$ & $0.07 \pm 0.11 \mathrm{ba}$ \\
90 & $21.00 \pm 8.13 \mathrm{~b}$ & $1.34 \pm 0.40 \mathrm{~cd}$ & $2.94 \pm 0.46 \mathrm{c}$ & $0.82 \pm 0.10 \mathrm{a}$ & $0.07 \pm 0.11 \mathrm{ba}$ \\
105 & $32.55 \pm 9.14 \mathrm{a}$ & $1.19 \pm 0.28 \mathrm{~d}$ & $3.82 \pm 0.69 \mathrm{~b}$ & $0.74 \pm 0.06 \mathrm{~b}$ & $0.06 \pm 0.07 \mathrm{bc}$ \\
\hline
\end{tabular}

Fuente: elaboración propia con base en los análisis.

Nota: $D D S$ = días después de la siembra, RAS = relación biomasa parte aérea y parte subterránea, ID = índice de calidad de Dickson, $\mathrm{AF}=$ área foliar, $\mathrm{RAR}=$ relación altura y longitud de la raíz, $\mathrm{IE}=$ índice de esbeltez. Letras distintas en las columnas son estadísticamente diferentes (Duncan, 0.05). Se incluyen valores promedio \pm desviación estándar $(n=9)$.

\section{CUADRO 4}

Área foliar e índices de calidad de planta en Leucaena esculenta a diferentes edades

\begin{tabular}{|llllll|}
\hline Árbol & RAS & ID & AF $\left(\mathrm{cm}^{2}\right)$ & RAR & IE \\
\hline 7 & $2.29 \pm 2.19 \mathrm{a}$ & $3.94 \pm 1.79 \mathrm{ba}$ & $15.53 \pm 12.69 \mathrm{ba}$ & $0.82 \pm 0.15 \mathrm{a}$ & $0.07 \pm 0.17 \mathrm{ba}$ \\
1 & $2.58 \pm 2.36 \mathrm{~b}$ & $3.68 \pm 2.05 \mathrm{~b}$ & $12.35 \pm 12.03 \mathrm{bc}$ & $0.72 \pm 0.18 \mathrm{~b}$ & $0.07 \pm 0.1 \mathrm{ba}$ \\
4 & $1.74 \pm 0.84 \mathrm{a}$ & $3.59 \pm 0.85 \mathrm{~b}$ & $12.54 \pm 10.68 \mathrm{bc}$ & $0.70 \pm 0.19 \mathrm{~b}$ & $0.07 \pm 0.14 \mathrm{~b}$ \\
2 & $1.77 \pm 1.19 \mathrm{a}$ & $3.21 \pm 0.97 \mathrm{~b}$ & $14.45 \pm 9.46 \mathrm{ba}$ & $0.67 \pm 0.10 \mathrm{cb}$ & $0.07 \pm 0.22 \mathrm{~b}$ \\
3 & $2.19 \pm 1.33 \mathrm{a}$ & $3.42 \pm 1.07 \mathrm{~b}$ & $14.27 \pm 14.84 \mathrm{ba}$ & $0.62 \pm 0.15 \mathrm{~cd}$ & $0.06 \pm 0.12 \mathrm{~b}$ \\
9 & $2.51 \pm 2.34 \mathrm{cb}$ & $3.67 \pm 1.60 \mathrm{~b}$ & $16.60 \pm 13.26 \mathrm{a}$ & $0.61 \pm 0.14 \mathrm{~cd}$ & $0.06 \pm 0.12 \mathrm{~b}$ \\
8 & $1.65 \pm 0.82 \mathrm{a}$ & $3.37 \pm 0.67 \mathrm{~b}$ & $14.08 \pm 12.62 \mathrm{ba}$ & $0.61 \pm 0.18 \mathrm{~cd}$ & $0.07 \pm 0.39 \mathrm{a}$ \\
6 & $3.22 \pm 3.28 \mathrm{a}$ & $4.54 \pm 3.03 \mathrm{a}$ & $9.95 \pm 9.94 \mathrm{c}$ & $0.65 \pm 0.15 \mathrm{cbd}$ & $0.06 \pm 0.12 \mathrm{~b}$ \\
5 & $1.87 \pm 1.11 \mathrm{a}$ & $3.20 \pm 1.02 \mathrm{~b}$ & $13.47 \pm 14.07 \mathrm{ba}$ & $0.59 \pm 0.13 \mathrm{~d}$ & $0.06 \pm 0.14 \mathrm{~b}$ \\
\hline
\end{tabular}

Nota: Promedio \pm desviación estándar, AF = área foliar, RAS = relación biomasa parte aérea y parte subterránea, ID = índice de calidad de dickson, $\mathrm{RAR}=$ relación altura y relación longitud de la raíz, $\mathrm{IE}=$ índice de esbeltez, ${ }^{*}=$ Letras diferentes en las columnas son estadísticamente diferentes (Duncan, 0.05). Los valores son promedios de los nueve árboles por muestreo.

Fuente: elaboración propia con base en los análisis. 


\section{Conclusiones}

Las variables morfológicas altura, diámetro de cuello, longitud de raíz y área foliar, así como los índices de calidad de planta de las progenies de Leucaena esculenta mostraron magnitudes diferenciables entre progenies, entre edad y entre la interacción de ambos factores. La progenie del árbol 7 obtuvo mayor crecimiento reflejado en los índices morfológicos. Las progenies de los árboles 7 y 4 formaron mayor cantidad de biomasa total $0.53 \pm 0.16$ y 0.51 $\pm 0.16 \mathrm{~g}$ /árbol, siendo las hojas el órgano con más biomasa que el tallo y raíz. Las plantas que presentan partes aéreas y radicales de mayor tamaño, pero con una conformación equilibrada y una gran capacidad de formación de nuevas raíces tienden a presentar mejores niveles de supervivencia.

\section{Prospectiva}

Los sistemas agroforestales de ladera de los Valles Centrales de Oaxaca, México, así como los existentes en otras áreas similares en condiciones ambientales y de sitio deben establecerse a través de una selección rigurosa de los árboles semilleros. La selección apropiada de fenotipos vigorosos, con diámetros al pie $>20 \mathrm{~cm}$, altura entre 2 y $3 \mathrm{~m}$, copa amplia y bifurcación a $1 \mathrm{~m}$ generan gran cantidad de vainas y semillas, así como progenies de calidad (Ferrer et al., 2003). Los índices de calidad evaluados permiten una selección apropiada de plántulas de Leucaena esculenta, donde las tasas de crecimiento en las variables morfológicas son proporcionales; esto genera plantas resistentes y vigorosas, características deseables en los sistemas agroforestales de la zona estudiada (Guevara y Guenni, 2007).

\section{REFERENCIAS}

Birchler, T., Rose, R. W., Royo, A. y Pardos, M. (1998). La planta ideal: revisión del concepto, parámetros definitorios e implementación práctica. Investigación Agropecuaria, 7(1-2), 109-121.

Chave, J., Riéra, B., \& Dubois, M. A. (2001). Estimation of biomass in a neotropical forest of French Guiana: Spatial and temporal variability. Journal of Tropical Ecology, 1, 79-96.

Escobar-Sandoval, M. C., Vargas-Hernández, J., López-Upton, J., S. Espinosa-Zaragoza y Borja-de la Rosa, A. (2018). Parámetros genéticos de calidad de madera, crecimiento y ramificación en Pinus patula. Madera y Bosques, 24(2), 1-11. https://doi.org/10.21829/myb.2018.2421595

Ferrer, Y., Sánchez, M., Rojas, R., Ramírez, V. M., Viloria, J. y Mendoza, S. (2003). Influencia de la escarificación y la imbibición sobre la germinación y crecimiento de plántulas de Leucaena leucocephala y Pithecellobium dulce. XV Congreso Venezolano de Botánica. Venezuela: Universidad de los Andes.

García, M. A. (2007). Importancia de la calidad del plantín forestal. XXII Jornadas Forestales de entre Ríos. Área Forestal de la EEA Concordia del INTA. Disponible en http://www.inta.gov.ar/concordia/info/ Forestales/contenido/pdf/2007/312.II. GARCIA.pdf

González, M. E., Donoso, C. y Escobar, B. (1996). Efecto de distintos regímenes de manejo radicular en el crecimiento de plantas de raulí (Nothofagus alpina (Poepp. et Endl) Oerst.) 1-0 a raíz desnuda. Bosque, 17, 29-41.

González, Y., Reino, J. y Machado, R. (2009). Dormancia y tratamientos pregerminativos en las semillas de Leucaena spp. cosechadas en suelo ácido. Pastos y Forrajes, 32, 1-1.

Guevara, E. y Guenni, O. (2007). Potencial de crecimiento de cuatro lineas de Leucaena leucocephala (Lam) de Wit durante el establecimiento. Perú: Sitio Argentino de Producción Animal. APPA-ALPA-Cusco.

Gutiérrez, B. (2003). Mejoramiento genético y conservación de recursos forestales nativos de Chile. Investigación agraria. Sistemas y recursos forestales, 12(3), 145-153. 
Haase, D. L. (2007). Morphological and physiological evaluations of seedling quality. In L. E. Riley, R. K. Dumroese, T. D. Landis (coords.). National proceedings: Forest and Conservation Nursery Associations. USA: SDA Forest Service. Fort Collins, CO.

ISTA (International Seed Testing Association). (2007). International rules for seed testing edition 2007. Switzerland. Jardel, P. E. (2006). Viejos y nuevos problemas en el sector forestal en México. Instituto Manantlán de Ecología y Conservación de la Biodiversidad. Centro Universitario de la Costa Sur. Auatlán de Navarro: Universidad de Guanajuato.

Kozlowski, T. T., \& Pallardy, S. G. (2002). Acclimation and adaptative response of woody plants to environmental stresses. Botanical Review, 68, 270. https://doi.org/10.1663/0006-8101(2002)068[0270: AAAROW] 2.0.CO;2

Mallén-Rivera, C. (2014). Recursos genéticos forestales. Revista Mexicana de Ciencias Forestales, 5(22), 4-9.

Medina, M. G., García, D. E., Clavero, T. e Iglesias, J. M. (2007). Estudio comparativo Moringa oleifera y Leucaena leucocephala durante la germinación y la etapa inicial de crecimiento. Zootecnia Tropical, 25, 83-93.

Medina, M. G., García, D. E., Moratinos, P. y Cova, L. J. (2011). Comparación de tres leguminosas arbóreas sembradas en un sustrato alcalino durante el periodo de aviveramiento. I. Variables morfoestructurales. Pastos y Forrajes, 34, 1-11.

Navarro, M. (2003). Desempeño fisiológico de las semillas de árboles de uso múltiple en el trópico. Pastosy Forrajes, 26, 97.

Noguera-Talavera, A., Reyes-Sánchez, N., José-Membreño, J., Duarte-Aguilar, C. y Mendieta-Araica, B. (2016). Calidad de plántulas de tres especies forrajeras (Moringa oleifera Lam., Leucaena leucocephala y Cajanus cajan) en condiciones de vivero. La Calera, 14, 21-27.

Olalde, G. V. M., Sánchez, P. G., Tijerina., L. C., Mastache, A. A. L. y Carreño, E. R. (2000). Crecimiento y distribución de biomasa en girasol en función del nitrógeno y densidad de población en clima cálido. Terra Latinoamericana, 18, 313-323.

Orozco, G. G., Muñoz, F. H. J., Villaseñor R. F. J., Rueda S. A., Sigala, R. J. A. y Prieto, R. J. A. (2010). Diagnóstico de calidad de planta en los viveros forestales del estado de Colima. Folleto Técnico Núm. 1. SAGARPA. INIFAP. CIRPAC. Michoacán: Campo Experimental.

Ortiz, M. E., Acosta H., C. C., Linares M., P., Morales R., Z. y Rebolledo-Camacho, V. (2016). Selección de árboles semilleros de Juglans pyriformis Liebm. en poblaciones naturales de Coatepec y Coacoatzintla, Veracruz. Revista Mexicana de Ciencias Forestales, 7(38), 43-58.

Poulsen, K. (2000). Calidad de la semilla. Concepto, medición y métodos para incrementar la calidad, en Técnicas para la germinación de semillas forestales. Serie Técnica. Manual Técnico 39. Turrialba: CATIEPROSEFOR-DFSC.

Prieto, R. J. A., García, R. J. L., Mejía, B. J. M., Huchín, A. S. y Aguilar, V. J. L. (2009). Producción de planta del género Pinus en vivero en clima templado frío. Publicación Especial Núm. 28. Durango: Campo Experimental Valle del Guadiana INIFAP-SAGARPA. Durango.

Prieto, R. J. A., Vera, C. G. y Merlín, B. E. (2003). Factores que influyen en la calidad de brinzales y criterios para su evaluación en vivero. Folleto Técnico Núm. 12. Durango: Campo Experimental Valle del Guadiana-INIFAPSAGARPA.

Ramírez, C. A. y Rodríguez, T. D. A. (2004). Efecto de la calidad de planta, exposición y micrositio en una plantación de Quercus rugosa. Revista Chapingo Serie Ciencias Forestales y del Ambiente, 10(1), 5-11.

Ramírez, C. J. A., Zapata, C. M. D., León, J. D. P. y González, M. I. H. (2007). Caída de hojarasca y retorno de nutrientes en bosques montanos andinos de Piedras Blancas, Antioquia, Colombia. Interciencia, 32, 303-311. 
Rodríguez, T. D. A. (2008). Indicadores de calidad de planta forestal. México: Universidad Autónoma Chapingo.

Rodríguez-Ortiz, G., Aldrete, A., González-Hernández, V. A., De Los Santos-Posadas, H. M., Gómez-Guerrero, A. y Fierros-González, A. M. (2011). ¿Afectan los aclareos la acumulación de biomasa aérea en una plantación de Pinus patula? Agrociencia, 45, 719-732.

Ruiz, T. E., Feble, G., Castillo, E., Jordan, H., Crespo, G., Valencia, N. y Díaz, J. (2007). La experiencia cubana en la agronomia y manejo de Leucaena leucocephala. La Habana: Instituto de Ciencia Animal. Disponible en http//www.cipav.org.co/redagrofor/memorias99/RuizTE2.htm

Sáenz, J. T. R., Villaseñor, F. J. R., Muñoz, H. J. F., Rueda, S. A. y Prieto, J. A. R. (2010). Calidad de plantas en viveros forestales de clima templado en Michoacán. Folleto Técnico No.17. Michoacán: Instituto de Investigaciones Forestales, Agrícolas y Pecuarias.

Sánchez, C., Montilla, E., Rivera, R. y Cupull, R. (2005). Comportamiento de 15 cepas de hongos micorrizógenos (HMA) sobre el desarrollo de posturas de cafeto en un suelo pardo gleyzoso. Revista Forestal Latinoamericana, 38, 83-95.

Sánchez, P. Y. y Ramírez-Villalobos, P. (2006). Tratamientos pregerminativos en semillas de Leucaena leucocephala (Lam.) de Wit. y Prosopis juliflora (Sw.) DC. Revista Facultad de Agronomia (LUZ), 23, 257-272.

Santiago, O., Sánchez, T., Monsalvo, V., Monroy R. C. y García S. G. (2007). Manual deproducción de especiesforestales tropicales en contenedor. Folleto Técnico Núm. 44. Veracruz: INIFAP. CIRGOC. Campo Experimental El Palmar.

SAS Institute Inc. (2004). SAS/STAT ${ }^{\circledR} 9.4$ User’s Guide. Cary, N. C.: SAS Institute Inc.

Singh, B., \& Singh, G. (2006). Effects of controlled irrigation on water potential, nitrogen uptake and biomass production in Dalbergia sissoo seedlings. Environment Experimental Botany, 55, 209-219.

Thompson, B. (1985). Seedling morphological evaluation. What can you tell by looking. In Evaluating seeling quality: Principles, procedures and predective abilities of major test. M. L. Durges. Forest Research Laboratory. Oregon State University.

Toral, I. M. (1997). Conceptos de calidad de plantas en viveros forestales. Ciclo económico forestal. Programa de Desarrollo Forestal Integral de Jalisco. Documento Técnico 1. Guadalajara.

Torres, A., Alvarado, A., Chacón, E., Zerpa, A. y Romero, R. (2002). Producción de semillas de Leucaena leucocephala. (Lam.) de Wit en Venezuela. Conferencia para el III Cursillo: El uso de recursos alimenticios para la producción de bovinos a pastoreo. Memorias XI Congreso Venezolano de Producción e Industria Animal. Venezuela.

Viveros-Viveros, H., Sáenz Romero, C., Vargas Hernández, J. y López Upton, J. (2006). Variación entre procedencias de Pinus pseudostrobus establecidas en dos sitios en Michoacán, México. Revista Fitotecnia Mexicana, 29(2), 121-126.

Willan, R. L. (2000). Pretratamientos de semillas, en Técnicas para la germinación de semillas forestales. Serie Técnica. Manual Técnico No. 39. CATIE-PROFESOR-DFSC. Turrialba.

Wu, F., Bao, W., Li, F., \& Wu, N. (2008). Effects of drought stress and N supply on the growth, biomass partitioning and water use efficiency of Sophora davidii Kom. ex. Pavol. seedlings. Environmental Experimental Botany, 63, 248-255.

Wullschleger, S., Meinzer, F. C., \& Vertessy, R. A. (2005). A review of whole-plant water use studies in trees. Tree Physiology, 18, 499-512.

Zobel, B. J. y Talbert, J. T. (1988). Técnicas de mejoramiento genético de árboles forestales. México: Limusa. 